\title{
Preprogramming Complex Hydrogel Responses using Enzymatic Reaction Networks
}

\author{
Sjoerd G. J. Postma, Ilia N. Vialshin, Casper Y. Gerritsen, Min Bao, and Wilhelm T. S. Huck*
}

\begin{abstract}
The creation of adaptive matter is heavily inspired by biological systems. However, it remains challenging to design complex material responses that are governed by reaction networks, which lie at the heart of cellular complexity. The main reason for this slow progress is the lack of a general strategy to integrate reaction networks with materials. Herein we use a systematic approach to preprogram the response of a hydrogel to a trigger, in this case the enzyme trypsin, which activates a reaction network embedded within the hydrogel. A full characterization of all the kinetic rate constants in the system enabled the construction of a computational model, which predicted different hydrogel responses depending on the input concentration of the trigger. The results of the simulation are in good agreement with experimental findings. Our methodology can be used to design new, adaptive materials of which the properties are governed by reaction networks of arbitrary complexity.
\end{abstract}

$L$ Living systems are adaptive and use enzymatic reaction networks to detect changes in their environment, process input information, and determine an appropriate response. ${ }^{[1]}$ Materials science has recently taken a keen interest in the adaptivity of living systems, ${ }^{[2]}$ and has created new materials with life-like properties such as self-healing, ${ }^{[3]}$ camouflaging, ${ }^{[4]}$ and control over surface characteristics. ${ }^{[5]}$ Impressive examples include the incorporation of the oscillating BelousovZhabotinsky reaction into a self-walking gel, ${ }^{[6]}$ and the work of Aizenberg and co-workers, ${ }^{[7]}$ who used chemo-mechanicochemical feedback loops to produce a homeostatic material. Others have pioneered control over hydrogel lifetimes with preprogrammed feedback loops using organic ${ }^{[8]}$ or enzymatic ${ }^{[9]}$ reactions.

However, progress towards "life-like" materials has been slow as we lack a general framework for constructing materials with autonomous behavior and preprogrammed responses to external stimuli. Designing materials with complex responses requires the incorporation of chemical reaction networks, where the kinetics within the system are suitably balanced. ${ }^{[10]}$ Here, we present a systematic approach to program the complex response of hydrogels, inspired by our previous work on enzymatic reaction networks. ${ }^{[11,12]}$ First, we developed a polyacrylamide (PAAm)-based hydrogel that

[*] S. G. J. Postma, I. N. Vialshin, C. Y. Gerritsen, M. Bao, Prof. W. T. S. Huck

Radboud University, Institute for Molecules and Materials Heyendaalseweg 135, 6525 AJ Nijmegen (The Netherlands) E-mail:w.huck@science.ru.nl

(9) Supporting information for this article can be found under: http://dx.doi.org/10.1002/anie.201610875. contains two orthogonal types of crosslinks that can be degraded or formed, respectively, through enzymatic activity (Figure 1A). When a trigger, the endopeptidase trypsin (Tr), is applied to the gel, degradation of the initial crosslinks 1 (C1) proceeds rapidly. Simultaneously, a crosslink precursor (a copolymerized thioester) is slowly cleaved, creating thiols that very quickly react with an added linker to form new crosslinks 2 (C2). Thus, a gel-liquid-gel transition takes place and a new gel is formed with potentially different properties such as shape and stiffness as compared to the initial gel.

Next, we introduced an enzymatic reaction network into the hydrogel of which the components all have their own specific function. When all components are present in the right concentrations, the network is able to sense the input concentration of $\mathrm{Tr}$, and determine the corresponding hydrogel response.

Importantly, the kinetics in the network are fully characterized, and the programmed response of the network is predicted by a computational model that is in good agreement with our experimental results. In this way, we provide a systematic approach for integrating reaction networks within adaptive materials.

We synthesized PAAm gels with two orthogonal crosslinkers (Figure 1 A): C1 is susceptible to cleavage by Tr, which will be used as a trigger. Tr also triggers the cleavage of thioester $\mathbf{3}$, revealing the cryptic thiol groups, which can react with the poly(ethylene glycol)-bis-maleimide crosslinker 4 ( $\mathrm{MW}=2000 \mathrm{~g} \mathrm{~mol}^{-1}$ ) that is present in the gel, forming C2 $\mathrm{Tr}$ rapidly cleaves amide bonds at the C-terminal end of positively charged amino acids, and therefore we synthesized C1 with an arginine-serine moiety in the middle of the molecule (Figure 1A; see section S2 of the Supporting Information (SI) for complete details on molecular structures and synthesis of all molecules used). We measured a value for $k_{\text {cat }} / K_{\mathrm{M}}$ (a measure of catalytic efficiency) of $\gg 24600 \mathrm{mM}^{-1} \mathrm{~h}^{-1}$ for Tr cleaving C1, confirming that Tr will rapidly degrade gels containing C1 (details of all kinetic studies are in section S3 of the SI).

For the cryptic crosslink precursor, we studied a number of amino acids that could serve as Tr-cleavable protecting groups of thiol side groups. Interestingly, due to the relatively high reactivity of thioesters, we found that the reactivity of $\mathrm{Tr}$ towards lysine thioesters was as high as the arginine amide bonds in $\mathrm{C} 1\left(k_{\text {cat }} / K_{\mathrm{M}}>29400 \mathrm{~mm}^{-1} \mathrm{~h}^{-1}\right)$. Therefore, the less reactive leucine thioester 3 was prepared (Tr hydrolysis $k_{\text {cat }} /$ $K_{\mathrm{M}}=575 \mathrm{~mm}^{-1} \mathrm{~h}^{-1}$ ). Importantly, thioester $\mathbf{3}$ is also quickly cleaved by another enzyme, chymotrypsin $\left(\mathrm{Cr} ; k_{\text {cat }} / K_{\mathrm{M}}=\right.$ $34400 \mathrm{~mm}^{-1} \mathrm{~h}^{-1}$ ), enabling more complex responses when using both enzymes in an enzymatic reaction network as we will show below. 


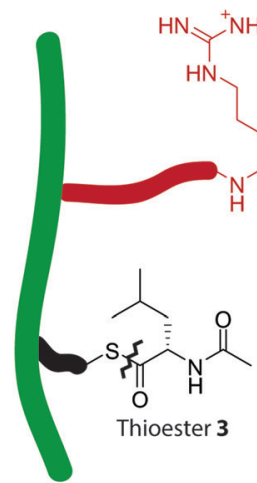

B

Gel (soaked with linker)

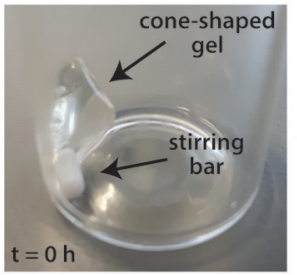

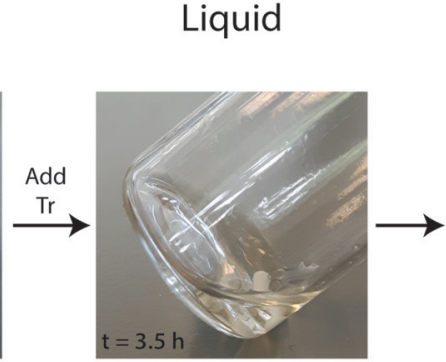

Liquid

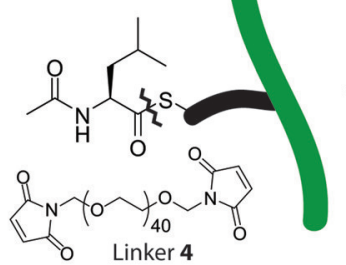

o Linker 4
Gel

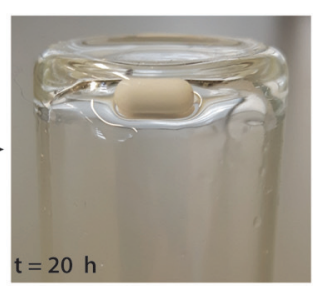

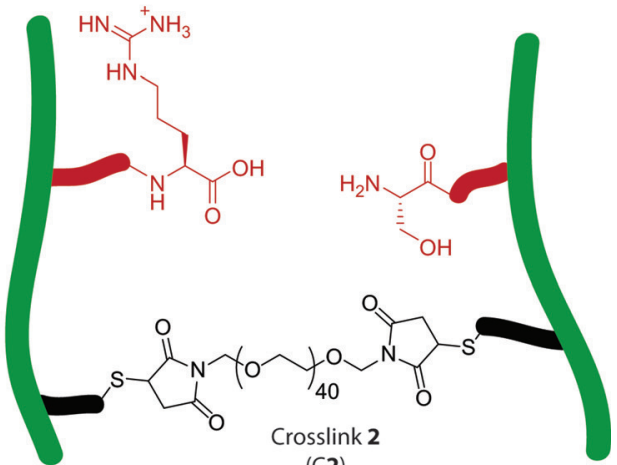

(C2)

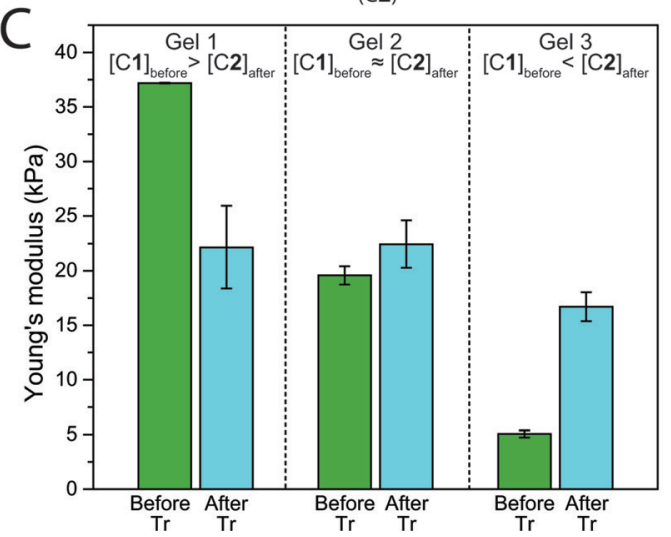

Figure 1. Gel-liquid-gel transitions. a) The initial polyacrylamide hydrogel contains crosslinks $\mathbf{1}$ (C1) and a copolymerized thioester $\mathbf{3}$. $\mathrm{C} \mathbf{1}$ is quickly degraded by trypsin ( $\mathrm{Tr}$ ), and thioester 3 is cleaved slowly by $\mathrm{Tr}$ or quickly by chymotrypsin (Cr) to produce thiols which subsequently form new crosslinks 2 (C2) after reacting with a linker (poly (ethylene glycol)-bis-maleimide). Zigzag lines indicate the sites of enzymatic cleavage. b) A cone-shaped gel $\left.(200 \mu \mathrm{L} \text { volume) containing } \mathrm{C} 1 \text { and thioester } 3 \text { is soaked with linker } \mathbf{4} \text {, after which Tr is applied ([Tr }]_{0}=5 \mu \mathrm{M}\right)$. A gel-liquid-gel transition occurs during which the shape of the gel changes, and the magnetic stirring bar (that plays no further role in this process) is encapsulated. c) Gels 1-3 contain different amounts of $\mathrm{C} 1$ and thioester 3, and consequently have different initial stiffness, but similar mechanical properties after treatment with Tr. Error bars indicate the range of Young's modulus in duplicate experiments (the error bar of gel 1 before application of $\mathrm{Tr}$ is very small). Measurements were performed by atomic force microscopy (see SI for details). All experiments are performed in $100 \mathrm{~mm}$ Tris- $\mathrm{HCl}$ buffer $\mathrm{pH} 7.5$ with $20 \mathrm{~mm} \mathrm{CaCl}_{2}$.

During C2 formation, the enzymatic cleavage of thioester should be the rate-limiting step to ensure control over the kinetics of gel formation by changing enzyme concentration. Therefore, the thiols formed after cleavage of the thioester should react as rapidly as possible with a crosslinker. Rate studies showed that maleimides react almost instantaneously with thiols in a Michael addition (section S3.3 of the SI). For that reason, the maleimide-containing linker $\mathbf{4}$ was chosen as the crosslinker to form $\mathrm{C} 2$ in all further studies.

Next, a gel was prepared that contained $\mathrm{C} \mathbf{1}$ and copolymerized thioester 3 (see Table S2 of the SI for the compositions of all gels made), and the hydrogel was soaked in a solution of linker 4 . When Tr was applied to the hydrogel $\left([\operatorname{Tr}]_{0}=5 \mu \mathrm{M}\right)$, the gel turned into a liquid after 3.5 hours (Figure 1B). The liquid formed a gel again, but with a different shape, after another 16.5 hours, which confirms that crosslink degradation and formation take place at convenient timescales.

The gel-liquid-gel transition transforms the mechanical properties of the gel, as the number of crosslinks $\mathrm{C} \mathbf{1}$ and $\mathrm{C} 2$ are independent of each other. We prepared three different gels soaked with linker $\mathbf{4}$ in which the amount of $\mathrm{C} \mathbf{1}$ in the initial gel was larger (gel 1), equal (gel 2) or smaller (gel 3) than the amount of $\mathbf{C} 2$ in the final gel (see Table S2 of the SI for gel compositions). The stiffness of the gels before and after triggering the rearrangement of the crosslinks was measured by atomic force microscopy coupled to a confocal microscope (see section S4 of the SI). Figure 1C shows the development of Young's moduli upon addition of $\mathrm{Tr}$ and waiting overnight. Initial moduli of $37.2 \mathrm{kPa}, 19.5 \mathrm{kPa}$, and $5.1 \mathrm{kPa}$ were obtained for gels $1-3$, respectively, which changed to $22.4 \mathrm{kPa}$ for both gels 1 and 2 , and $17.0 \mathrm{kPa}$ for gel 3 after the crosslink rearrangement. Gel 1 had become softer, gel 2 had retained its stiffness, and gel 3 had turned into a stiffer gel. Thus, it was shown that three mechanically different hydrogels can obtain a similar stiffness upon treatment with Tr.

Next, we program the activity of Tr by incorporating the enzyme in a reaction network. Figure $2 \mathrm{~A}$ shows how Tr can be formed autocatalytically from its own precursor trypsinogen $(\mathrm{Tg})$, creating a positive feedback loop. This amplification of [ $\mathrm{Tr}]$ is expected to speed up both the degradation of $\mathbf{C} \mathbf{1}$ and the activation of $\mathrm{C} 2$. Tr also rapidly converts chymotrypsinogen $(\mathrm{Cg})$ into its active form chymotrypsin $(\mathrm{Cr})$ with $k_{\text {cat }} /$ 
A

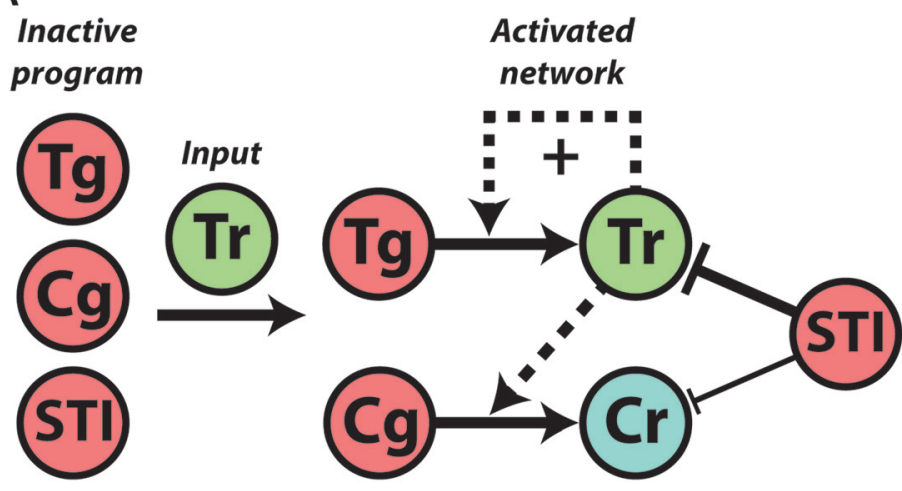

C
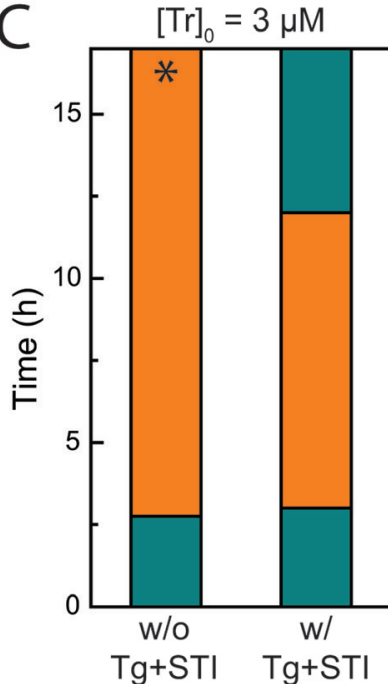

$[\mathrm{Tr}]_{0}=8 \mu \mathrm{M}$

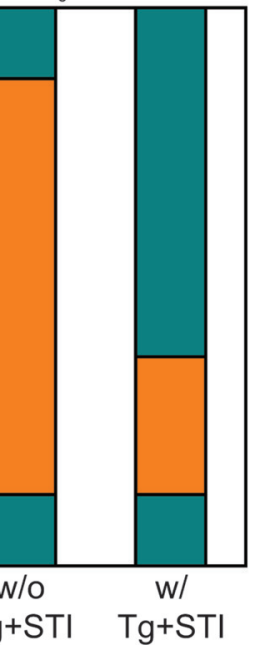

B

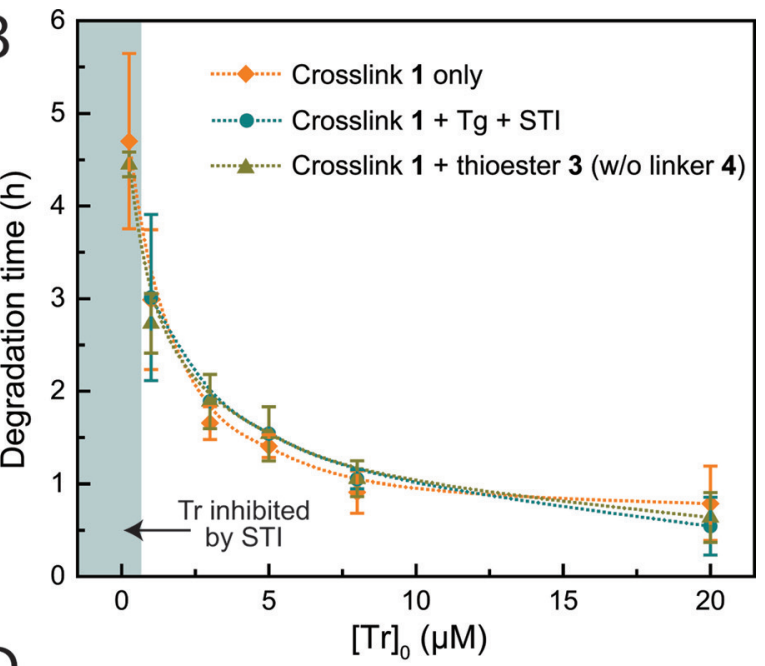

Gel

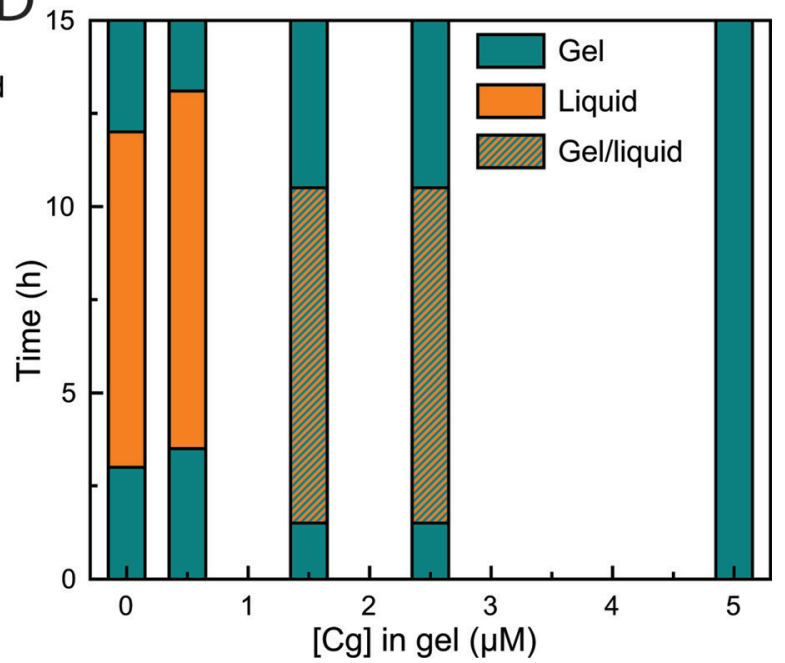

Figure 2. Introducing an enzymatic reaction network in the hydrogel. a) Schematic representation of the network. Trypsin (Tr) catalyzes its own formation by activating trypsinogen ( $\mathrm{Tg}$ ), creating a positive feedback loop. $\mathrm{Tr}$ also activates chymotrypsinogen (Cg) to form chymotrypsin (Cr). Soybean trypsin inhibitor (STI) inhibits $\mathrm{Tr}$ and $\mathrm{Cr}$ strongly and weakly, respectively (indicated by the thickness of the inhibition arrows). b) The degradation time of different gels in vial tests is dependent on the concentration of $\operatorname{Tr}\left([\operatorname{Tr}]_{0}=0.25-20 \mu \mathrm{M}\right.$. For the cyan curve: $[\mathrm{Tg}]_{0}=80 \mu \mathrm{M}$, $[\mathrm{STI}]=1.9 \mu \mathrm{M})$. In the cyan region, Tr is fully inhibited by STI. Error bars are standard deviations obtained from triplicate experiments. c) Influence of $\mathrm{Tg}$ on the rate of gel reformation $\left([\mathrm{Tg}]_{0}=80 \mu \mathrm{M},[\mathrm{STI}]=1.9 \mu \mathrm{M}\right.$, if present) in vial tests. The asterisk in the left column indicates that a gel was formed again after 80 hours, which is off the scale. d) Influence of $[\mathrm{Cg}]$ on the rate of $\mathrm{C} 2$ formation in vial tests (in all gels: $[\mathrm{Tg}]_{0}=80 \mu \mathrm{M}$, $[\mathrm{STI}]=1.9 \mu \mathrm{M}$, and $[\mathrm{Tr}]_{0}=3 \mu \mathrm{M}$ ). For the gel/liquid regions (orange and cyan stripes), the contents of the vial were difficult to classify as either being a soft gel or a viscous liquid. All experiments are performed in $100 \mathrm{~mm}$ Tris- $\mathrm{HCl}$ buffer pH 7.5 with $20 \mathrm{~mm} \mathrm{CaCl}$.

$K_{\mathrm{M}}=12500 \mathrm{~mm}^{-1} \mathrm{~h}^{-1}$. Cr does not cleave C1 (Figure S21 of the SI), but rapidly cleaves thioester $\mathbf{3}$, activating the formation of $\mathbf{C 2}$. Finally, the enzymatic network is deactivated by the presence of soybean trypsin inhibitor (STI) protein, a strong, reversible inhibitor of $\operatorname{Tr}\left(K_{\mathrm{D}} 1 \mathrm{nM}\right)^{[12 \mathrm{a}]}$ that sets a threshold for the Tr input that will activate the network. We found that STI also reversibly inhibits $\mathrm{Cr}\left(K_{\mathrm{D}}=634 \mathrm{nM}\right.$, Figure S14), but with a dissociation constant at least two orders of magnitude larger than for Tr.

Then, we gradually increased the complexity of the preprogrammed system to integrate the reaction network within the hydrogels in a step-by-step approach. First, we tested the degradation rate of a gel only containing $\mathrm{C} \mathbf{1}$ crosslinks using various $[\operatorname{Tr}]_{0}(0.25-20 \mu \mathrm{M}$, orange diamonds in Figure $2 \mathrm{~B}$ ). We found a nonlinear correlation between $[\operatorname{Tr}]_{0}$ and the time required for a gel-liquid transition to occur, with average gel degradation times ranging between 47 minutes and 4.7 hours. Then, we prepared C1-only gels, but soaked these in $\operatorname{Tg}(80 \mu \mathrm{M})$ and STI $(1.9 \mu \mathrm{M})$, and again used the same range of $[\operatorname{Tr}]_{0}$ to trigger degradation (cyan circles, Figure 2B). Now, we observe a similar trend in gel degradation times as in the previous experiment, but below a certain input threshold (cyan region in Figure 2B), Tr is fully inhibited by STI and the gel does not degrade, even when left for more than a week.

In addition, when gels containing both $\mathrm{C} \mathbf{1}$ and thioester $\mathbf{3}$ were prepared without network components or linker 4 (so no gel reformation could occur), we did not see any significant difference from the previous experiments $\left([\operatorname{Tr}]_{0}=0.25-20 \mu \mathrm{M}\right.$, green triangles, Figure 2B). This observation confirms that $\mathrm{Tr}$ 
cleaves $\mathbf{C} \mathbf{1}$ much faster than thioester $\mathbf{3}$, and when the two are combined in one hydrogel, Tr will first degrade most of $\mathbf{C} \mathbf{1}$ crosslinks before significant activation of the cryptic crosslink sites occurs.

In Figure $2 \mathrm{C}$, the influence of $\mathrm{Tg}$ on the rate of gel reformation is shown. In this case, gels containing both $\mathrm{C} \mathbf{1}$ and thioester $\mathbf{3}$ were prepared, and soaked with linker $\mathbf{4}$ to enable gel reformation. The gel reformation time decreased from 80 hours (indicated by an asterisk in Figure 2C) in the absence of Tg and STI to 12 hours in the presence of both proteins (80 and $1.9 \mu \mathrm{M}$, respectively) when $[\operatorname{Tr}]_{0}$ was $3 \mu \mathrm{M}$. The influence of the positive feedback was less pronounced at a higher $[\operatorname{Tr}]_{0}(8 \mu \mathrm{M})$ applied to the gel, but still the gel reformation time decreased 2.5 times in the presence of $\mathrm{Tg}$ and STI. Interestingly, we do not observe an influence of the positive feedback on the gel degradation time, indicating that again $\mathrm{C} 1$ cleavage is dominant over other processes catalyzed by $\mathrm{Tr}$ at the early stages after application of Tr.

Finally, C1- and thioester 3-containing gels were soaked in solutions with varying concentrations of $\mathrm{Cg}$ in the presence of $\operatorname{Tg}(80 \mu \mathrm{M})$ and STI $(1.9 \mu \mathrm{M})$, and in all cases $[\operatorname{Tr}]_{0}$ was $3 \mu \mathrm{M}$ (Figure 2D). The observed response of the gel was highly dependent on $\mathrm{Cg}$ concentration. At $0.5 \mu \mathrm{M} \mathrm{Cg}$, gel-liquid-gel transitions were still observed, but at concentrations of 1.5 and $2.5 \mu \mathrm{M} \mathrm{Cg}$ the hydrogel went through a gel/liquid phase in which it was difficult to classify the material as either a soft gel or a viscous liquid (indicated by orange/cyan stripes in Figure 2D). Increasing the concentration of $\mathrm{Cg}$ even further led to gel-gel transitions without an intermediate liquid state, because the rate of formation of $\mathbf{C} \mathbf{2}$ is greatly enhanced by the production of $\mathrm{Cr}$ and ultimately outpaces the degradation of C1.

In the previous experiments, we have seen three different gel responses: 1) inhibition of Tr by STI, 2) gel-gel transitions when the rate of $\mathbf{C} \mathbf{1}$ degradation is slower than of $\mathbf{C} 2$ formation, and 3) gel-liquid-gel transitions when C1 degradation is faster than $\mathbf{C} 2$ formation. As our final demonstration of the ability to preprogram the complex response of the hydrogel materials, we coupled the different hydrogel responses to the input $\operatorname{Tr}$ concentration. A computational model in MATLAB was constructed using all the determined rate constants in ordinary differential equations (see section S5 of the SI), to explore the hydrogel response at different initial concentrations of $\mathrm{Cg}$ and $\mathrm{Tr}$.

In Figure 3, the phase diagram generated by these simulations is depicted, in which three regions corresponding to the three different gel responses are observed. Importantly, the phase diagram shows that the strength of Tr trigger can lead to any of the three responses if the concentration of $\mathrm{Cg}$ exceeds $0.5 \mu \mathrm{M}$. We verified these computational results in experiments using identical gels $([\mathrm{Cg}]=1.5 \mu \mathrm{M},[\mathrm{Tg}]=80 \mu \mathrm{M}$, $[\mathrm{STI}]=1.9 \mu \mathrm{M})$, and we indeed obtained different responses depending on the $\operatorname{Tr}$ concentration (squares in Figure 3, pictures and procedures in section S4.1.7 of the SI). Our model is in good agreement with the experimental results, although the influence of STI is slightly overestimated in the simulations. Additional modelling indicated that we indeed need the full reaction network to obtain the complex gel responses (Figure S26 and S27).

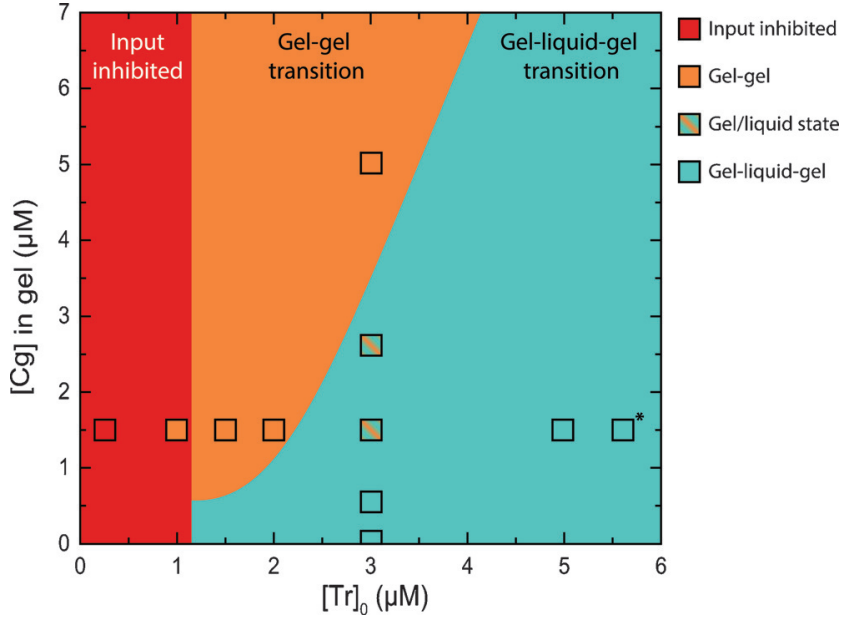

Figure 3. Phase diagram of $[\mathrm{Cg}]$ vs. $[\mathrm{Tr}]_{0}$ obtained by mathematical modelling. Experiments are shown in squares (in all cases $[\mathrm{Tg}]_{0}=80 \mu \mathrm{M}$ and $\left.[\mathrm{STI}]=1.9 \mu \mathrm{M}\right)$. The blue square with an asterisk denotes experiments conducted at $[\mathrm{Tr}]_{0}=7,20$, or $50 \mu \mathrm{M}$, all of which show gel-liquid-gel transitions. $\mathrm{Cg}=$ chymotrypsinogen, $\mathrm{Tr}=$ trypsin.

In short, we have shown a stepwise design of hydrogel materials with preprogammable responses to environmental triggers (in this case [Tr]). The incorporation of a small reaction network that is coupled to the crosslinking chemistry in the hydrogels can be used to create materials that sense and process a biochemical input. We believe that this approach can be extended further to create adaptive matter based on hydrogels containing more complex reaction networks that can control the time profile of the chemical response within the material. In addition, the methodology can be applied to other networks based on DNA, ${ }^{[13]}$ or small organic molecules. $^{[14]}$

\section{Acknowledgements}

We thank Simon Yang and Hugo Veldhuizen for their contributions to the synthesis of thioester $\mathbf{3}$ and lysine thioester, respectively. This work was supported by funding from the Dutch Ministry of Education, Culture and Science (Gravity programme 024.001.035), and the Radboud Nanomedicine Alliance.

\section{Conflict of interest}

The authors declare no conflict of interest.

Keywords: enzymes · gels · life-like systems programmable materials reaction networks

How to cite: Angew. Chem. Int. Ed. 2017, 56, 1794-1798 Angew. Chem. 2017, 129, 1820-1824

[1] a) J. E. Purvis, G. Lahav, Cell 2006, 152, 945-956; b) B. N. Kholodenko, Nat. Rev. Mol. Cell Biol. 2006, 7, 165-176; c) D. Bray, Nature 1995, 376, 307-312. 
[2] a) M. A. Cohen Stuart et al., Nat. Mater. 2010, 9, 101-113; b) B. A. Grzybowski, W. T. S. Huck, Nat. Nanotechnol. 2016, 11 , 585-592.

[3] a) S. R. White, N. R. Sottos, P. H. Geubelle, J. S. Moore, M. R. Kessler, S. R. Sriram, E. N. Brown, S. Viswanathan, Nature 2000, 409, 794 - 797; b) P. Cordier, F. Tournilhac, C. Soulié-Ziakovic, L. Leibler, Nature 2008, 451, 977-980; c) T.-S. Wong, S. H. Kang, S. K. Y. Tang, E. J. Smythe, B. D. Hatton, A. Grinthal, J. Aizenberg, Nature 2012, 477, $443-447$.

[4] S. A. Morin, R. F. Shepherd, S. W. Kwok, A. A. Stokes, A. Nemiroski, G. M. Whitesides, Science 2012, 337, 828-832.

[5] a) X. Yao, Y. Hu, A. Grinthal, T.-S. Wong, L. Mahadevan, J. Aizenberg, Nat. Mater. 2013, 12, 529-534; b) K.-C. Park, P. Kim, A. Grinthal, N. He, D. Fox, J. C. Weaver, J. Aizenberg, Nature 2016, $531,78-82$.

[6] S. Maeda, Y. Hara, T. Sakai, R. Yoshida, S. Hashimoto, $A d v$. Mater. 2007, 19, 3480-3484.

[7] X. He, M. Aizenberg, O. Kuksenok, L. D. Zarzar, A. Shastri, A. C. Balazs, J. Aizenberg, Nature 2012, 487, 214-218.

[8] a) J. Boekhoven, A. M. Brizard, K. N. K. Kowlgi, G. J. M. Koper, R. Eelkema, J. H. van Esch, Angew. Chem. Int. Ed. 2010, 49, 4825-4828; Angew. Chem. 2010, 122, 4935-4938; b) J. Boekhoven, W. E. Hendriksen, G. J. M. Koper, R. Eelkema, J. H. van Esch, Science 2015, 349, 1075-1079.

[9] a) S. Debnath, S. Roy, R. V. Ulijn, J. Am. Chem. Soc. 2013, 135, 16789-16792; b) C. G. Pappas, I. R. Sasselli, R. V. Ulijn, Angew. Chem. Int. Ed. 2015, 54, 8119-8123; Angew. Chem. 2015, 127,
8237-8241; c) T. Heuser, E. Weyandt, A. Walther, Angew. Chem. Int. Ed. 2015, 54, 13258-13262; Angew. Chem. 2015, 127, 13456-13460; d) E. Jee, T. Bansagi, Jr., A. F. Taylor, J. A. Pojman, Angew. Chem. Int. Ed. 2016, 55, 2127-2131; Angew. Chem. 2016, 128, 2167-2171.

[10] L. Heinen, A. Walther, Soft Matter 2015, 11, 7857-7866.

[11] a) S. N. Semenov, A. S. Y. Wong, R. M. van der Made, S. G. J. Postma, J. Groen, H. W. H. van Roekel, T. F. A. de Greef, W. T. S. Huck, Nat. Chem. 2015, 7, 160-165; b) A. S. Y. Wong, S. G. J. Postma, I. N. Vialshin, S. N. Semenov, W. T. S. Huck, J. Am. Chem. Soc. 2015, 137, 12415-12420.

[12] a) S. N. Semenov, A. J. Markvoort, W. B. L. Gevers, A. Piruska, T. F. A. de Greef, W. T. S. Huck, Biophys. J. 2013, 105, $1057-$ 1066; b) S. N. Semenov, A. J. Markvoort, T. F. A. de Greef, W. T. S. Huck, Angew. Chem. Int. Ed. 2014, 53, 8066-8069; Angew. Chem. 2014, 126, 8204-8207.

[13] a) J. Stricker, S. Cookson, M. R. Bennett, W. H. Mather, L. S. Tsimring, J. Hasty, Nature 2008, 456, 516-519; b) A. Padirac, T. Fujii, A. Estévez-Torres, Y. Rondelez, J. Am. Chem. Soc. 2013, 135, 14586-14592.

[14] S. N. Semenov, L. J. Kraft, A. Ainla, M. Zhao, M. Baghbanzadeh, V. E. Campbell, K. Kang, J. M. Fox, G. M. Whitesides, Nature 2016, $537,656-660$.

Manuscript received: November 7, 2016

Final Article published: January 10, 2017 